\title{
DISTAL ARTHROGRYPOSIS MULTIPLEX CONGENITA WITH MULTISYSTEM INVOLVEMENT: A CASE REPORT
}

\author{
Shilpa Kantamneni1, Sujay Kumar Earan², Arulkumaran Arunagirinathan³, Priyaja Paramasivam ${ }^{4}$ \\ ${ }_{1}^{1}$ Post Graduate Student, Department of Paediatrics, SMVMCH, Puducherry. \\ ${ }^{2}$ Assistant Professor, Department of Paediatrics, SMVMCH, Puducherry. \\ 3Professor, Department of Paediatrics, SMVMCH, Puducherry. \\ ${ }^{4}$ Post Graduate Student, Department of Paediatrics, SMVMCH, Puducherry.
}

\section{ABSTRACT}

Arthrogryposis Multiplex Congenita (AMC) is an uncommon but easily recognizable syndrome of musculoskeletal system. All four extremities and the trunk may be affected. The hallmark of this entity is the lack of active and passive motion in the affected extremities. Rather than a specific diagnosis, it is a clinical finding. The earliest antenatal diagnosis of arthrogryposis can be made as early as 7 to 8 weeks. Treatment options include multidisciplinary approach with careful orthopaedic care followed by physiotherapy. In this case report of a newborn with distal AMC with multisystem involvement based on literature and clinical experience, the authors present an update on aetiology, antenatal diagnosis, classification and management of AMC. Through this report, we would like to stress upon the significant role of antenatal ultrasound in not only diagnosing but also in prevention of this condition.

\section{KEYWORDS}

Arthrogryposis, Contracture, Ultrasonography, Pregnancy, Amyoplasia, Distal Arthrogryposis.

HOW TO CITE THIS ARTICLE: Kantamneni S, Earan SK, Arunagirinathan A, et al. Distal arthrogryposis multiplex congenita with multisystem involvement: a case report. J. Evolution Med. Dent. Sci. 2016;5(62):4397-4399, DOI: 10.14260/jemds/2016/1004

\section{INTRODUCTION}

Arthrogryposis Multiplex Congenita (AMC) is an uncommon, but easily recognizable syndrome of musculoskeletal system. All four extremities and the trunk may be affected. The hallmark of this entity is the lack of active and passive motion in the affected extremities. In addition they may have either clubfeet or vertical talus, both of which are more resistant to treatment than those not associated with arthrogryposis.[1]

It is non-progressive in nature and has a multifactorial aetiology with an incidence of 1:3000 live births, equal in gender ratio. ${ }^{[1]}$ Rather than a specific diagnosis, it is a clinical finding. It is found to be connected with 300 different disorders. In this case report, we present a newborn with distal AMC with multisystem involvement, which was missed during the routine antenatal scan. Through this report, we would like to stress upon the significant role of antenatal ultrasound in not only diagnosing but also in prevention of this condition.

\section{CASE REPORT}

An 11 days old female child, second by birth order, born to a couple of second degree consanguineous marriage, hailing from Cuddalore, a rural district in South India, was brought with complaints of multiple deformity of lower limbs since birth.

The previous child was born as a preterm with extreme low birth weight with absent toes and fingers. The baby expired at 30 days of life, the cause of death was not evaluated further for the child.

Financial or Other, Competing Interest: None.

Submission 16-06-2016, Peer Review 21-07-2016,

Acceptance 27-07-2016, Published 04-08-2016.

Corresponding Author:

Dr. Shilpa Kantamneni,

Post Graduate Student

Paediatrics, SMVMCH,

Puducherry,

E-mail: shilpa.kantamneni@gmail.com

DOI: 10.14260/jemds/2016/1004
Mother was on regular antenatal care, an anomaly scan was taken at 5 months, although no abnormalities were detected at that time. Antenatal period was uneventful.

The child was born at full term by elective caesarean section in view of previous LSCS. Baby cried immediately after birth. Birth weight was $2.7 \mathrm{~kg}$, total length $43 \mathrm{cms}$, head circumference $33.2 \mathrm{cms}$. The child was noted to have deformities of both the lower limbs and was referred to our hospital for further evaluation.

On musculoskeletal survey, b/l knee contractures were noted along with $\mathrm{b} / \mathrm{l}$ ankle ramus deformity, syndactyly was noted in the right foot, marked talipes equinovarus deformity was noted in both the ankles (Figure 1 and 2). The radiological findings can be noted in Figure 3.

Range of movements of right lower limb: hip flexion of 0 $85^{\circ}$, hip extension of $0-8^{\circ}$, knee flexion of $0-70^{\circ}$, and knee extension $0-5^{\circ}$.

In the left lower limb the baby was able to flex the hip from $0-85^{\circ}$, hip extension of $0-10^{\circ}$, knee flexion of $0-25^{\circ}$, knee extension of $0-5^{\circ}$ was noted.

No obvious facial dysmorphism was noted, external genitalia female, no obvious abnormality was noted in skull and spine.

2D Echocardiography revealed congenital acyanotic heart disease. Large ostium secundum type of ASD with left to right shunt and a small perimembranous VSD with moderate mitral regurgitation without significant pulmonary hypertension.

Ultrasound of the abdomen revealed bilateral hydronephrosis and gall bladder was not visualised. Ultrasonogram of the skull was found to be normal. 

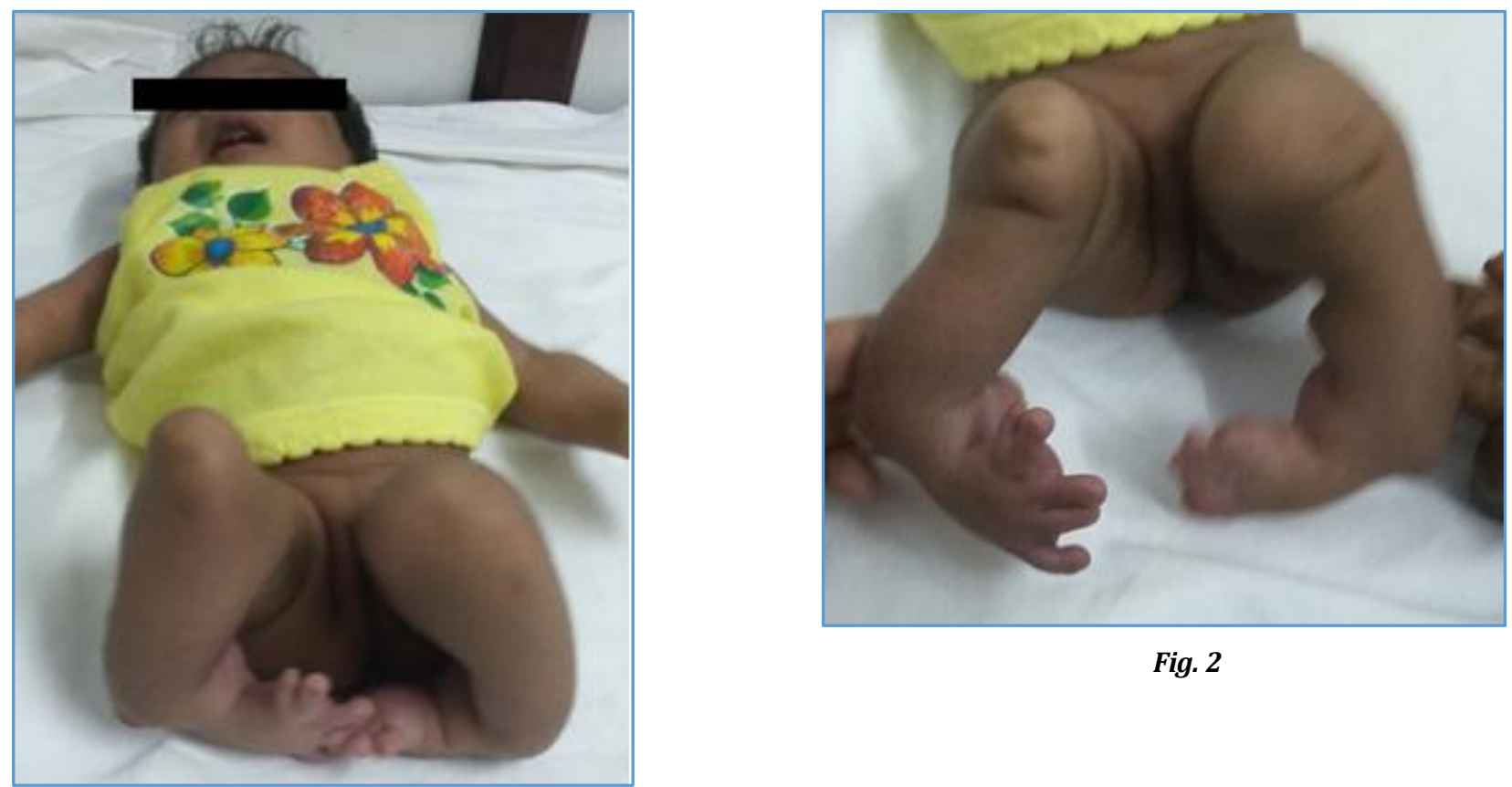

Fig. 2

Fig. 1

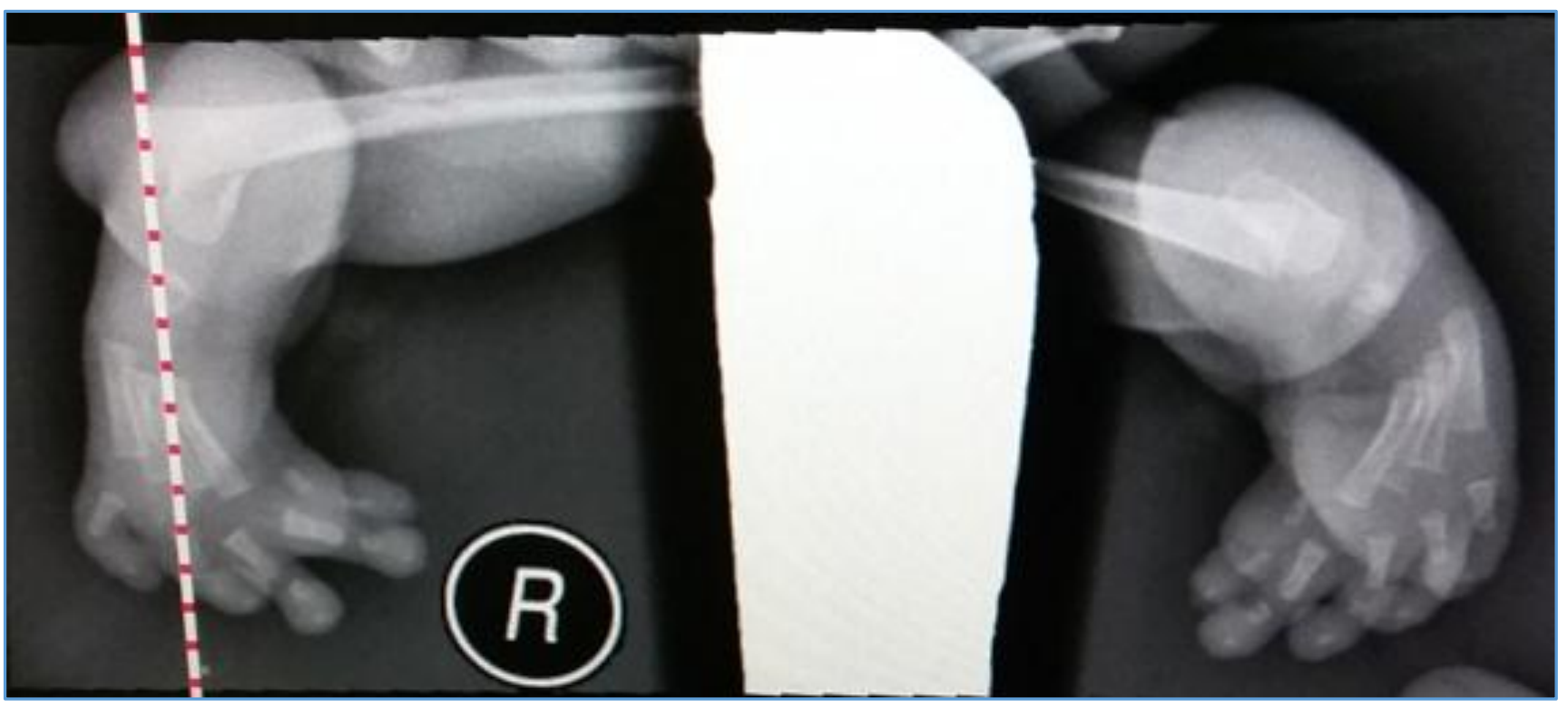

Fig. 3

\section{DISCUSSION}

Arthrogryposis Multiplex Congenita (AMC) is a disorder with multiple aetiological factors. The underlying cause is predominantly due to chromosomal aneuploidy followed by anterior horn cell disease and peripheral neuropathies. The second most common cause is muscle abnormalities; this is proved by the significant connection between AMC and myasthenia gravis. The pathogenesis in this condition is transplacental shift of maternal antibodies into the foetal circulation, which acts against the acetylcholine receptors in the foetus, thereby inhibiting their function and causing injury to foetal muscle, thereby causing weakened foetal movements in utero and development of contractures. Foetal myopathy due to sarcomeric protein dysfunction can cause distal arthrogryposis.[2]
Connective tissue disorders might result in AMC by restoring the lost muscle mass by forming collagen, which thickens at the region of joint capsule and causes joint fixation consequently causing decreased fetal movements and contractures.

The other intrauterine causes include intrauterine space constraint which is usually seen in multiple pregnancies, oligohydramnios, uterine deformities, fibroids, amniocentesis at 15 weeks of gestational age, which shows 10 to 15 fold increase in development of contractures. This theory that abnormal intrauterine pressure was concerned in the production of arthrogryposis was initially observed by Denis Browne.[3] It was reported in his paper titled "Joint contractures in children" as early as 1956.(4) Intrauterine vascular compromise too may lead to contractures. 
Drugs like curare which is a muscle relaxant, misoprostol, cocaine and alcohol are associated with this condition.

Syndromes which are associated with arthrogryposis are Pierre Robin, Moebius syndrome, Arnold-Chiari syndrome, Prune belly syndrome, Meckel-Gruber syndrome, Zellweger syndrome.[5]

The clinical classification according to the degree of severity ("Munich classification").

Type 1: Primary affection of the extremities, possibly neck and trunk muscles.

Primary affection of hands and feet; part of this is the contractural arachnodactyly (So called distal arthrogryposis; autosomal dominant).

Affection of all extremities including shoulder and hip joint (60-80\%) with symmetrical internal rotation of the shoulders, fixed extended elbows, flexion and extension contractures of the knee and ankle joints, talipes equinovarus (Approx. 85\%) (So called amyoplasia; in most cases sporadical).

Type 2: Primarily midline malformations; affections of extremities (Vide type 1) as well as malformations of different organs (e.g. diaphragmatic hernia, pronounced scoliosis), pterygium.

Type 3: Further dysmorphic disorders and malformations; disorders of the CNS.[6]

The earliest prenatal diagnosis of arthrogryposis can be made as early as 7 to 8 weeks. The earliest indication by ultrasonography being reduced foetal movements as well as abnormal position of the foetus.[5] The other ultrasound findings include fixed flexion deformity, micrognathia, oligohydramnios, limb deformities, cerebral ventriculomegaly, dysmorphic features.

Increased nuchal translucency at 10 to 14 weeks or increased nuchal translucency along with scoliosis at 15 weeks of gestational age also signifies arthrogryposis multiplex congenita. In our case, antenatal scan was normal with no evidence of above said signs.

The disease might finally end up with serious limb deformities, short stature, kyphoscoliosis and multiple fracture.[7] Treatment options include multidisciplinary approach with careful orthopaedic care followed by physiotherapy. In the initial five years of life, respiratory infection is in the major hazard to life. ${ }^{[8]}$ If these children survive the first five years, the expectation is that they will reach maturity to find a place in the community. Operative correction plays a much larger role in the treatment of affected lower limbs. Three out of four children with lower limb involvement can be enabled to walk if the need for repeated operation is accepted.(8) Distal Arthrogryposis (DA) syndromes are often hereditary and joint involvement is predominantly in the hands and feet.[2] Genetic counselling plays a significant role in the prevention of this condition.[9]

The intent of reporting this case is to stress upon the importance of antenatal ultrasonography in not only diagnosing this condition, but also its prevention. We also would like to stress upon the lack of a reliable and competent ultrasound in the rural and remote parts of India. This has not only lead to the increased mortality and morbidity of the patient, but also increases economic and social burden on the parents.

\section{REFERENCES}

1. Avery GB. Avery's neonatology: pathophysiology and management of the newborn. In: MacDonald MG, Seshia MM, Mullett MD, eds. $6^{\text {th }}$ ed. Philadelphia: Lippincott Williams \& Wilkins 2005.

2. Kimber E. AMC: amyoplasia and distal arthrogryposis. Journal of Children's Orthopaedics 2015;9(6):427-32.

3. Mackenzie DY. Arthrogryposis multiplex congenita. Proceedings of the Royal Society of Medicine 1959;52(12):1101-5.

4. Discussion on joint contractures. Proceedings of the Royal Society of Medicine 1957;50(2):69-74.

5. Kalampokas E, Kalampokas T, Sofoudis C, et al. Diagnosing arthrogryposis multiplex congenita: a review. ISRN Obstetrics And Gynecology, Article ID 264918 2012;2012:6.

6. Oppitz F, Speulda E. Anaesthesia recommendations in patients suffering from arthrogryposis multiplex congenita. Orpha.net. [cited 31 May 2016, 2011]. Available from: http://www.orpha.net.

7. Mishra NR, Panda PC, Arun KM, et al. Bruck syndrome: a rare case report with dextrocardia and meningomyelocele. Journal of Global Biosciences 2014;3(7):1021-4.

8. Gibson DA, Urs ND. Arthrogryposis multiplex congenita. J Bone Joint Surg Br 1970;52(3):483-93.

9. Niveditha R, Singhal D, Keshav M, et al. Arthrogryposis multiplex congenita. Karnataka Paediatric Journal 2011;25(3):91-3. 